\title{
Mathematical tools to understand the field theories of the Standard Model and beyond
}

\author{
Philippe Durand \\ CNAM- Paris, \\ département Mathématiques-Statistiques \\ Email: philippe.durand@ lecnam.net
}

Received: June 15, 2021. Revised: July 19, 2021. Accepted: July 23, 2021. Published: July 28, 2021.

\begin{abstract}
Since Isaac Newton the understanding of the physical world is more and more complex. The Euclidean space of three dimensions, independent of time is replaced in Enstein's vision by the Lorentzian space-time at first, then by four dimensions manifold to unify space and matter. String theorists add to space more dimensions to make their theory consistent. Complex topological invariants which characterize different kind of spaces are developed. Space is discretized at the quantum scale in the loop quantum gravity theory. A non-commutative and spectral geometry is defined from the theory of operator algebra by Alain Connes. In this review, our goal is to enumerate different approaches implementing algebra and topology in order to understand the standard model of particles and beyond.
\end{abstract}

Keywords: NC-geometry, Supersymmetry, Heat kernel, Loop Quantum Gravity

\section{INTRODUCTION}

The unification of physical theory remains an unattainable question. The first experiments about gravity, electricity, or magnetism were static theory all defined in space and universal time.They have given way to dynamical theories and unified electromagnetism with Maxwell's equations, Newton theory is replaced by special, and general relativity. The concept of forces lead to the concept of fields, of symmetry. A fundamental point is that the introduction of time in special or general relativity as a variable in the same way as the other space variables, In static theory, we have an universal time while in dynamic theories the time (proper time) is coupled to the other space variables. in different theories, time has not same meaning. The notion of invariance relatively to a gauge group, or by diffeomorphism in relativity lead to the introduction of different mathematical theories in the physical world, group theory, and Lie algebras, Riemannian geometry, algebra or topology. The fundamental problem is unification at the quantum level of two incompatible theories; Large-scale gravitation and quantum mechanics. The first success was the unification through the standard model of electromagnetism of weak and strong nuclear interactions. The standard model of particles $(U(1) \times S U(2) \times S U(3))$ composed of bosons (interactions) and fermions (particles) perfectly describe world at quantum scales. In this review article, we want to discuss on different strategies to understand the role that gravity can play at the quantum level.

The first, string theory, was originally an hadronic theory describing interactions (bosonic theory). Assuming the existence of supersymmetric particle and changing length scale of string (planck order $10^{-33} \mathrm{~cm}$, we obtain a theory allowing to construct a particle of spin 2 which seems to describe a quantification of gravitation: the graviton. Another contribution of supersymmetry is to allow the entry into the scene of topology in field theory: The index theorem shows that a good strategy is not the study of all the spectrum of an operator but rather the state of vacuum (ground state). Around this concept of index of an operator, topology, ground state, Witten describes a new QFT: topological quantum field theory. In this context, one can define different theories of fields, with boundary, associated with the pairs (open strings, branes), (describing the standard model), or without boundary (closed strings) describing the gravity.

A second approach to understand gravitation at the quantum scale is to be directly inspired by Heisenberg's ideas of quantum mechanics: the notion of spectral lines of a chemical element such as the hydrogen atom leads to describe, from the point of view of classical mechanics, the convolution group, algebra of frequencies emitted: these structures are commutative. However, in quantum reality, the convolutional algebra must be replaced by matrix algebra and therefore is non-commutative. This fundamental idea led Alain Connes [15] to be interested in the algebra of operators and to construct non-commutative geometry (NCG).

The last approach is to describe some elements of another theory: loop quantum gravity. For a long time people tried to overcome the problems linked to the perturbative approach in gravitation. The perturbative approach produces infiny quantities in Feynman diagram so is not renormalizable. The string theory solve this by replacing Feynman diagrams by Riemann surfaces with boundary. The LQG proposes to avoid the perturbative approach but at the cost of a complex mathematical language.This theory adopt Hamiltonian language (as previously NCG), to quantify gravity. The basic idea amounts to saying that at the planck scale the geometry is discretized, The concept of holonomy ( as in RG to measure the curvature), is already present in the quantification of electromagnetism (Wilson loops). The concept plays a major role in quantum gravity hence The discretization at the planck scale of space time leads to the creation of Spin networks and spin foam managing the passage between authorized quantum states. 


\section{QUANTUM MECHANIC AND QUANTUM FIELD THEORY}

\section{A. Lagrangian approach}

The concept of field is fundamental in physics. A field $\varphi$ is a function of a source space (world sheet for string theory ) into a target space, $M$ with a sufficient number of dimensions. So this given a "package" $(\Sigma, M, \varphi)$ and a classical action: $S$ where: $\Sigma$ is the source space, often a manifold: for The classical mechanic of the point is the time axis (world line). For the conformal field theories like bosonic string theories: Riemann surface ...

Lagrangian density is a function on one or more fields and its first derivatives:

$$
\mathcal{L}=\mathcal{L}\left(\varphi_{1}, \varphi_{2}, \ldots, \partial_{\mu} \varphi_{1}, \partial_{\mu} \varphi_{2} \ldots\right)
$$

Classical action is the integral of the classical Lagrangian density on space $S=\int \mathcal{L} d^{n+1} x$

Principle of least action: The minimization of the action: ( $\delta S=0$, leads to each field noted just $\varphi$ to the Euler-Lagrange equation which gives the equations of motion of the particle

$$
\frac{\partial \mathcal{L}}{\partial \varphi}-\partial_{\mu}\left(\frac{\partial \mathcal{L}}{\partial\left(\partial_{\mu} \varphi\right)}\right)=0
$$

A quantization can be seen in through a classical action quantified from the path integral. We can then define the correlation functions dependent on selected observable. These functions depend, for example, the metric that is chosen on the target space.

Uncertainty on the position or momentum in quantum mechanic led to replace the classical solution (least action) by the partition function or the set of all possible solutions: It is the path integral [16]

$$
\mathcal{Z}=\int_{\Sigma \rightarrow M} e^{-S(\varphi)} \mathcal{D} \varphi
$$

Similarly, one can calculate correlation functions, or functions with $n$ marked points.

$$
<\varphi_{1}\left(x_{1}\right), \ldots, \varphi_{n}\left(x_{n}\right)>=\int_{\Sigma \rightarrow M} \varphi_{1}\left(x_{1}\right) \ldots \varphi_{n}\left(x_{n}\right) e^{-S(\varphi)} \mathcal{D} \varphi
$$

\section{B. Hamiltonian approach}

The Legendre transformation is an ingredient allowing to pass from Lagrangian formalism to Hamiltonian formalism. This makes it possible to set up non-perturbative methods in field theory: geometric quantification. Briefly we have a simplectic manifold $(M, \omega)$ endowed with a Hamiltonian $H_{t}$, a Poisson bracket. The Hamilton evolution equation describes the dynamic evolution of an observable by the Hamiltonian flow. The quantification consists in replacing this by a Hilbert space of states, by the Eisenberg operator and a Lie bracket.

\section{TOPOLOGICAL FIELD THEORY}

The first theory that we will visit in an attempt to understand gravity at the quantum level is supersymmetric string theory. It is a perturbative approach in which topology and the search of invariants play a big role. An essential idea to understand is that the not exited states, in quantum mechanic (ground states) describe the topology and by the same a kind of quantification.

\section{A. Standard model, String theory, and supersymmetry}

String theory was originally an hadronic theory to describe the strong nuclear interaction, it was a bosonic theory: it did not involve particles of matter (the fermions) but only the cement that unites them: the interactions between quarks. It is by accident that gravity came to play a preponderant role in this theory. The modification of the length of the strings on the scale Planck and the consideration of the supersymmetry allow to eliminated the tachyons (negatives probabilities) and reduced the number of additional dimensions of the theory. The consideration of supersymmetry staged the topology in quantum field theory. The first decisive step was to understand that the Atiyah and Singer index theorem: demonstrating the identity between the analytical index of certain elliptical operators and the topological index characterizing the topology of geometrical objects. It should also be noted that the supersymmetry can also be applied to other gauge theory: Chern-Simons, Yang mills theory. Supersymmetry tell us that each elementary particle (bosons or fermions) of the standard model is equipped with super-partners (respectively of integer and half integer spin)

\section{B. Index theorem}

We have reviewed in [17] some tools about index theory. By introducing supersymmetry to classical field theory, we make these theories topological, by adopting the perturbative Lagrangian approach, we can introduce the path integral and the correlation functions. In the latter, the points or functions of these points are replaced by cohomology classes and this makes it possible to define topological invariants;. Let us quickly recall the key points of the construction. The index of an elliptical pseudodifferential operator is constructed from cohomology classes. In the initial work of Atiyah [], This was also at the origin of a new theory the topological $\mathrm{K}$ Theory. It is rather the local theory of the index defined from the heat kernel that will allow the entry into the scene of supersymmetry.

Recall that in [] we have described that what ensures the existence and uniqueness of the differential system $\mathrm{L}(\mathrm{u})=$ $\mathrm{v}$, where $\mathrm{L}$ is an elliptic operator of a vector bundle $E$ in a vector bundle $F$ is controlled by 1 'analytical index of operator $L$ :

$$
\operatorname{Ind}_{a}(L)=\operatorname{dim}(\operatorname{ker}(L)-\operatorname{dim}(\operatorname{Coker}(L))
$$

The simplest model is the case where $L$ is a linear map between two spaces of finite dimensions $E$ and $F$. In this 
case it is immediate that the analytical index is equal to the topological index indeed:

$$
\begin{array}{r}
\operatorname{Ind}_{a}(L)=\operatorname{dim}(k e r(L)-\operatorname{dim}(\operatorname{Coker}(L))= \\
\operatorname{dim}(k e r(L)-(\operatorname{dim}(F)-\operatorname{dim}(\operatorname{Im}(L))= \\
\operatorname{dim}(E)-\operatorname{dim}(F)
\end{array}
$$

This last quantity is the difference between two dimensions of space it obviously has a topological nature, moreover a small deformation of the operator $\mathrm{L}$, being able to make it non-bijective for example, does not change the nature of the index. Always with this example we can introduce the spirit of supersymmetry:

\section{Supesymmetrie heat kenel}

by the previous example, $E=\mathbb{C}^{n}, F=\mathbb{C}^{m}$, we can now build a new space using the tensor product $E \otimes F$. If $f$ denote linear map between us with matrix $P$ and $f^{*}$ is. We can build a new space using the tensor product, in this, the matrix $\left(\begin{array}{cc}0_{n, n} & P^{*} \\ P & 0_{m, m}\end{array}\right)$ is an endomorphism that exchanges $E$ and $F$ we will say that $E$ is the space of Bosons, $F$ that of fermions. This endomorphism can be seen as a square root of endomorphism with matrix: $\left(\begin{array}{cc}P^{*} P & 0_{n, m} \\ 0_{m, n} & P P^{*}\end{array}\right)$ with, $P P^{*}$ and $P^{*} P$ : selfadjoint In physics, the first matrix denote the Dirac operator, and it is the square root of the second matrix: the Laplacian (Hamiltonian) The eigenvalues of $P P^{*}$ and $P^{*} P$ are positive (self-adjoint operator). It has two heat equations (bosonic aspect), easily resolvable:

$$
\begin{aligned}
& \left(\frac{d}{d t}+P^{*} P\right) u_{1}(t)=0 \\
& \left(\frac{d}{d t}+P P^{*}\right) u_{2}(t)=0
\end{aligned}
$$

there solutions are $u_{1}(t)=e^{-t P^{*} P} u_{1}(0)$ and $u_{1}(t)=$ $e^{-t P P^{*}} u_{2}(0)$

The non-zero eigenvalues of $P P^{*}$ and $P^{*} P$ are identical. they represent the exited states (in quantum mechanics). We show that the kernel of $P^{*} P$ (resp. $P^{*} P$ ) are those of $P$ (resp. $P^{*}$ ), They represent non-exited states or ground states.

We can then define the "super-trace" of $P^{2}$ :

$$
\begin{array}{r}
\operatorname{Str}\left(e^{-t P^{2}}\right)=\operatorname{Tr}\left(e^{-t P^{*} P}\right)-\operatorname{Tr}\left(e^{-t P P^{*}}\right)= \\
\operatorname{Tr}\left(e^{-t P}\right)-\operatorname{Tr}\left(e^{-t P^{*}}\right)= \\
\operatorname{dim}(\operatorname{ker}(f))-\operatorname{dim}(\operatorname{coker}(f))=\operatorname{Ind}_{a}(f)
\end{array}
$$

Solve the heat equation on $\left(E, P^{2}\right)$, gives the spectrum of the selfadjoint operator $P^{2}$; Let $P: E \rightarrow F, P^{*}$ be adjoint, the embedding of $\mathrm{E}$ in the super-space $E \otimes F$ gives a natural self-adjoint: $P P^{*}+P^{*} P$ whose in the square root, $P$ and its adjoint exchange $E$ (bosons) and $F$ (fermions). the index of $P$ select the ground states of the supersymmetric system so constructed.

We have seen a first equivalence: the index theorem connects a problem of analysis to a problem of topology. And now, In a supersymmetric world, only the non-exited states contribute to the super partition function of the quantum theory considered.
These ground states are the signatures of the topology, given by Index theorem. We have the "triangle" : Analysis-topologyPhysic

\section{ANALYSIS}

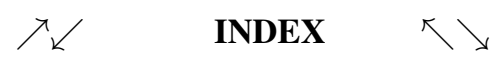

TOPOLOGY $\rightleftarrows \quad$ PHYSIC

A concrete realisation of previous construction in physic is ,given by:

\section{Application 1: Supersymmetric Quantum Mechanic}

1) Bosonic version: If we consider a particle moving on a manifold $\mathrm{M}$, the quantification of this problem consists in solving the Shrodinger equation. Mathematically it is to solve the heat equation on a manifold, the Laplacian $\Delta=-\frac{1}{2} \frac{d^{2}}{d \theta^{2}}$ is Hamiltonian $H$ in physic. For simplicity, take the circle $S^{1}$ of radius $\beta$. The solution of heat equation is here:

$$
\operatorname{Tr}\left(e^{-\beta H}\right)=\sum_{n=-\infty}^{+\infty} \exp \left(\frac{-\beta 2 \pi^{2} n^{2}}{R^{2}}\right)
$$

A physical interpretation of this quantity is the partition function $Z(\beta)$.

2) Supersymmetric version: Physicists in the mid-sixties postulate the existence of a new symmetry that Symmetry of Noether: this is supersymmetry, we shall return in more detail later. Briefly, in the standard model, there are two types of particles: the bosons that carry the interactions, and the fermions that constitute matter. Supersymmetry postulates that each fermion has a super-partner bosonic correspondent (and reciprocally). A boson has the vocation of being a switching variable, that is a pair variable, while a fermion must be anticommutative, we will say that it is an odd variable. That explains why we choose the algebra of the differential forms of degree 1 to construct the fermionic variables. The most suitable framework for introducing supersymmetry is that of Clifford algebras. The idea to add fermions artificially will be to inject algebras functions on a variety In the algebra of differential forms. The differential forms of even degrees will represent the bosons, while the differential forms of odd degrees will be the fermions. We then construct a so-called super-space, we often note $\psi$, or $\theta$ a fermionic variable. We denote by $\mathcal{Q}$ the operator BRST in mathematics is simply the differential of the De Rham complex. One can construct its adjoint noted $\overline{\mathcal{Q}}$. For example, for the circle $S^{1}$, to take into account supersymmetry, we replace the Hilbert space $\mathcal{H}\left(S^{1}, \mathbb{C}\right)$ by a larger space : $\mathcal{H}\left(\Omega^{*}\left(S^{1}\right) \otimes \mathbb{C}\right)$.

$$
\mathcal{H}_{\mathcal{B}}=\Omega_{0}\left(S^{1}\right) \stackrel{\mathcal{Q}, \overline{\mathcal{Q}}}{\longrightarrow} \mathcal{H}_{\mathcal{F}}=\Omega_{1}\left(S^{1}\right)
$$

The Dirac operator $D$ exchanges bosons and fermions and reciprocally in the case of the circle $S^{1}$, we simply have:

$$
D=\mathcal{Q}+\overline{\mathcal{Q}}
$$


This operator is a square root of the Laplacian, the semiLaplacian which restores the energy $H$ of the system:

$$
H=\frac{1}{2}(\mathcal{Q} \overline{\mathcal{Q}}+\overline{\mathcal{Q}} \mathcal{Q})=\frac{1}{2} \Delta
$$

In this context, the partition function on the circle $S^{1}$ becomes, the index:

$$
\begin{array}{r}
Z_{S}(\beta)=\operatorname{Str}\left(e^{-\beta H}\right)=(-1)^{F} \operatorname{Tr}\left(e^{-\beta H}\right)= \\
\operatorname{Tr}\left(e^{-\beta \overline{\mathcal{Q}} \mathcal{Q}}\right)-\operatorname{Tr}\left(e^{-\beta \mathcal{Q} \overline{\mathcal{Q}})}\right)
\end{array}
$$

The two operators $\mathcal{Q} \overline{\mathcal{Q}}, \overline{\mathcal{Q}} \mathcal{Q}$ have the same eigenvalues and on the other hand: the $\mathcal{Q} \overline{\mathcal{Q}}$ kernels are those of $\overline{\mathcal{Q}}$ and that of $\overline{\mathcal{Q}} \mathcal{Q}$ is the $\mathcal{Q}$; The preceding equality thus becomes:

$$
Z_{S}(\beta)=\operatorname{Str}\left(e^{-\beta H}\right)=\operatorname{dimKer}(\mathcal{Q})-\operatorname{dimker}(\overline{\mathcal{Q}})
$$

In the framework of Riemannian geometry, we know that each class of cohomology has a harmonic representation. $\Psi$ verifying $\mathcal{Q} \psi=\overline{\mathcal{Q}} \psi=0$ Is in the kernel of the Laplacian, which physically represents the states of the vacuum. In other words, when one is interested in the supersymmetric model, the function of partition depends only on the non-exited states: the state of the vacua of a theory. That is the topological side of the index of operator (here $D$ ): ind (D). In addition, the two kernels: $\operatorname{ker}(\overline{\mathcal{Q}} \mathcal{Q}), \operatorname{ker}(\mathcal{Q} \overline{\mathcal{Q}})$ represents the betti numbers: $b_{0}$ and $b_{1}$ respectively. So the index of the Dirac operator on $S^{1}$ vanish.

The above calculations can be generalized for any compact variety. The de Rham complex is exploited, the even differential forms represent the bosons, the odd forms, the fermions. We find by taking Dirac operator $d+d \dagger$ that the index of this operator on the manifold is the Euler Poincaré characteristic of the manifold.

We have given here a very simple construction of the supersymmetry: we can indeed construct the bosons and the fermion from a split on the de Rham cohomology (separating even and odd differential forms). The more natural constructions are made by using spinors that can be defined from Clifford algebras. We then define a kind of square root of geometry, a spinor is in a way the square racine of a vector Dirac operator the square root of a Laplacian ....

\section{E. Topological field theory}

Topological field theory exploits many complexes resulting from algebraic topology, many topological invariants can be calculated: by exploiting the theory of bundles and connections on a principal bundle, we can calculate: the Donaldson invariants [6], Seiberg-Witten, in dimension 4 , in dimension 3 the correlation functions lead exploit Wilson loops ChernSimons theory and provide new invariants for the theory of knots [22]. The exploitation of the moduli of stable curves in symplectic geometry leads to the Gromov-Witten invariants [11] ...
1) Construction of a topological field theory: Historically, we have seen that some field theory could be expressed in a purely topological way, for example the Chern-Simons theory. This field theory has a 3-dimensional variety as its starting point. ,. The correlation functions that we can define from this theory are based on topological observables, Wilson's loops, they have led to the definition of new invariants for knot theory. Another example, Yang-Mills theories can be made topological, if we consider the addition of supersymmetry. AtIyah and Segal [] are the first to have defined the bases of an axiomatics of topological field theory: They started from the notion of riemann surface in conformal field theory. A Riemann surface has good properties such as being able to be broken down into elementary bricks like a pair of pants. Theory is also at the origin of the definition of the sigma model. Witten proposes another approach. He wants to define a purely topological field theory, by making the correlation functions of a field theory independent of the notion of metric. One strategy to do this, is to assume the existence of supersymmetry: Noether's principles of symmetry are adapted by adding supersymmetric variables. That helped extend the BRST formalism for supersymmetric topological field theories. Calculations of correlation functions in which the points are replaced by cohomology classes, lead to define spaces of instantons: moduli space whose dimension calculated by the index theorem lead to enumerative geometry.

2) moduli space: An indispensable structure, on which modern invariant research is based, is moduli space. In the different applications, it should be properly defined. The custom wants it to be invariant under the action of a symmetry group (gauge group in physics). The moduli space is therefore obtained by quotienting a set of geometric objects: in the theories of Donaldson and Seiberg-Witten, it is a space of connections. For Seiberg-Witten invariants, the gauge group is $U(1)$. For those of Donaldson [6], we take the set of auto-dual connections (ASD) and the gauge group is $S U(2)$. For string theory, the modulus space is made from holomorphic curves [? ] and Cauchy Riemann conditions. For example, the modulus space allowing calculation of Seiberg-Witten invariants [9] is constructed as follows: $X$ is a manifold of dimension 4, oriented, endowed with a $\operatorname{Spin}^{c}$ structure characterized by the line bundle $\operatorname{det}\left(S^{+}\right)$where $S^{+}$is the bundle of positive spinors. we consider a connection $A$ on the principal bundle $U(1)$ then:

$\mathcal{M}_{L}=\left\{(\Phi, A) \in \Gamma\left(S^{+}\right) \times \mathcal{C}\left(P_{U(1)}\right): D_{A}=0, F_{A}^{+}=\frac{1}{4} \omega^{\Phi}\right\} / \mathcal{G}$

\section{Rules for the calculation of invariants}

1) Properly define the modulus space.

2) Make it compact to kill singularities.

3) Define an Elliptical complex in algebraic topology 
associated with this moduli space.

4) Using the index theorem or the Riemann-Roch theorem, Calculate the index of the elliptic complex thus constructed: This is the dimension of the moduli spaces.

5) In good cases, adequate correlation functions allow the dimension of the moduli space to be made zero.

6) define the invariants for example using the tools of enumerative geometry(in the case where moduli space has dimension zero.

\section{NON COMMUTATIVE GEOMETRY}

The approach of non-commutative geometry (NCG) has as its starting point the origins of mechanics and the study of the distribution of frequencies in chemical elements. Rydberg shows that the lines are distributed s according to the formula $\therefore$ On the other hand, if in the approach of classical mechanics, all the frequencies form a group, Experience proves that this is not the case at the quantum level. More precisely, the convolutional algebra associated with the groupoid $\triangle=\{(i, j), i, j \in$ $I\}$ with composition rule $(i, j)(j k)=(i, k)$ algebra =behaves like a matrix algebra. therefore a noncommutative algebra. It was Heisenberg who was the first to have the idea of ââreplacing the observable quantities of classical mechanics by "matrix mechanics" in which the observable quantities no longer commute. This naturally led Alain Connes, inventor of non-commutative geometry, to develop his vision of the quantum world. Taking into account the principles of geometric quantification, quantification in (NCG) will consist in algebraizing the geometric and topological structures in order to be able to couple the external geometry of a particle and its internal geometry as well as all the operators of differential calculus, of the theory measurement and algebraic topology:

\section{A. Tools of NCG}

The macroscopic space from which we are working is a Riemannian manifold $M$ (or rather Lorentzian of dimension 4). We further assume that $M$ is spinorial. A natural algebra which corresponds to it is the algebra of functions over $\mathbf{M}$ infinitely differentiable. We can then define the spinor bundle $S$ on this manifold as well as the Classical Dirac operator obtained as the square root of the Riemannian Laplacian. We need to define an Hilbert space, it will be the set of sections $L^{2}(M, S)$ of the bundle of spinors. This involves a triplet, the triplet $(\mathcal{A}, \mathcal{H}, D)$. The generalization can then be done by performing appropriate semidirect products of algebras, so as to combine all or part of the Lagrangian of the standard model and that of Relativity: $\mathcal{A}$ becomes a possibly noncommutative algebra, $\mathcal{H}$ a Hilbert space and D the associated Dirac operator. Consideration of differential geometry from this point of view allows non-trivial extensions of field theory. The strategy is to define increasingly complex triplets to include the internal geometry of particle physics.

\section{B. One example : Gravity coupled with standard model}

The symmetry group for particle of standard model is given by: $\mathcal{U}=\mathcal{C}^{\infty}\left(\mathbb{R}^{4}, U(1) \times S U(2) \times S U(3)\right)$ his also the local gauges transformations.

Lagrangian for standard model coupled with gravity is given by: $\mathcal{L}=\mathcal{L}_{g_{\mu \nu}}+\mathcal{L}_{\text {particlee }}$

The Triplet $(\mathcal{A}, \mathcal{H}, D)$ is given by:

$\mathcal{A}=\mathcal{C}^{\infty}\left(\mathbb{R}^{4}\right) \otimes\left(\mathbb{C} \oplus \mathbb{H} \oplus M_{3}(\mathbb{C})\right)$, where $\mathbb{H}$ is quatenions

$H=L^{2}\left(\mathbb{R}^{4}, \mathbb{H} \oplus \mathbb{H}\right) \otimes \mathbb{C}$ : the spinor bundle

$D=D_{g_{\mu \nu}} \otimes 1+\gamma_{5} \otimes D_{F}$ which combine gravitational Dirac and operator derived by standard model.

A decisive remark is that intern deformation of metric are parametized by Gauges Bosons. CThis work [] offers a remarkable prediction for the mass $M_{B H}$ of the Higgs boson: $M_{B H} \in[160 G E V, 180 G E V]$

\section{LOOP QUANTUM GRAVITY}

The quantification from the perturbative theory is a success of string theory. To do this, we replace the Feynman diagrams by Riemann surfaces with boundary. This is not the way adopted to quantify gravity by the LQG approach. The strategy adopted in this theory is to prepare the Hilbert-Einstein action so that it can facilitate the quantization. The notion of holonomy from kind of Wilson loops (as in Chern-Simons theory), and the concept of spin network play a great role.

\section{A. Introduction of $L Q G$}

The first step to implement the Loop Quantum Gravity is therefore to find a formalism which makes it compatible with Hamiltonian mechanics [21]. It is necessary to create a couple of variables "position, moment" which make it possible and apply the principles of geometric quantization. Recall that in the Hilbert-Einstein action, the field considered is the metric $g_{\mu \nu}$; This field does not allow quantization. Even the approximate attempts that proposing a slight perturbation of the flat metric: $g_{\mu \nu}=\eta_{\mu \nu}+h_{\mu \nu}$ cannot be renormalized.

Ashtekar [19] leads to rewrite the equation of general relativity in the language of Cartan. The new entity, which replaces the metric is the triad or mobile frame. It replaces the metric $g_{\mu \nu}$ by two variables which are the 1-form of connection and the 2-form of curvature (as we do in electromagnetism or quantum electrodynamics). In Einstein's time-space, the time and space variables are separated. This will stage two groups of gauge; $S O(3,1)$ manages the transformation of the four-dimensional space, while $S O(3)$, manages only three-dimensional space variables. In fact the spinor representation are the essential bricks to recreate the geometry (square root of geometrical tools), thus it stages the 2-folds cover of rotational gauge groups namely $S L(2, \mathbb{C})$ and $S U(2)$.

\section{B. Triangulated Space and space time}

Another essential point in this theory is that the authors do not have the ambition to manufacture an unified theory everything: they want just quantized space-time. This leads to the definition of a discrete geometry, which consists in triangulating space and space-time. This leads to the notion of 
spin network, dual graph of spatial triangulation (kinematics of the theory), and to the notion of spin foams (triangulation of space-time) dynamic aspect of the theory.

\section{Quantum mecanic for $L Q G$}

Firstly consider quantification of space: when we change the tetrahedron in the discrete geometry modeling the threedimensional space, this amounts to defining a discrete parallel transport and producing curvature. We use to do this the 1 form of connection defined from the gauge group $S U(2)$. Concretely, we can think that we have a discrete rotation in space. In the dual graph, each tetrahedron is a node. The passage from one tetrahedron to another is an edge of the graph. We will therefore define the hilbert space corresponding to an edge $e \curvearrowright e^{\prime}$ of the graph (that corresponds to tetrahedrom change)

1) Hilbert space at the edge: When looking at the space part, the interesting gauge group is $S U(2)$ : covering space of $S O(3)$. Recall that this is the set of unitary matrices with unit determinant:

$h=\left(\begin{array}{cc}a & -\bar{b} \\ b & \bar{a}\end{array}\right)$, with $|a|^{2}+|b|^{2}=1$

Geometricaly is the three sphere $S^{3}$ thus it is compact manifold. In the Hamiltonian formalism, what replaces the position variable to be quantified which is here a "rotation" given by an element of the associated spin group, namely $S U(2)$. then construct the Hilbert space: $\mathcal{H}=L^{2}(S U(2))$, the waves functions $\psi$ are summable square functions (for Harr measure) from $S U(2)$ to $\mathbb{C}$.

You can define a scalar product in $L^{2}(S U(2))$ given by:

$$
\langle\varphi, \psi\rangle=\int_{S U(2)} \overline{\psi(h)} \varphi(h) d h
$$

It is well known that the associated Lie-agebra is $s u(2)$ generated by the Pauli matrices:

$$
\sigma_{1}=\left(\begin{array}{cc}
0 & 1 \\
1 & 0
\end{array}\right), \sigma_{2}=\left(\begin{array}{cc}
0 & -i \\
i & 0
\end{array}\right), \sigma_{3}=\left(\begin{array}{cc}
1 & 0 \\
0 & -1
\end{array}\right)
$$

so $h=e^{-i \alpha^{i} \sigma_{i} / 2}=\cos \left(\frac{\alpha}{2)} \cdot 1+i \sin \left(\frac{\alpha}{2)} \frac{\alpha^{i}}{\alpha} \sigma_{i}\right.\right.$ with $\alpha=\sqrt{\alpha_{i} \alpha_{i}}$ We can consider left (and right) invariant vector fields $\vec{L}$ of component:

$$
L_{i} \psi(h)=\lim _{t \rightarrow 0}\left[\frac{\psi\left(h e^{-t \frac{i \sigma_{i}}{2}}\right)-\psi(h)}{t}\right]
$$

. The variables and conjugate moment of Hamiltonian mechanics are here the connection linked to an element of the group $S U(2)$ and the conjugate element is the derivation defined before (18). The latter allows to give again the metric: $G_{l k}=L_{l} . L_{k}$ which corresponds to the normal angle between two faces and the area of the triangle which connects two tetrahedra and satisfy:

$$
A^{2}=L_{l} \cdot L_{l}
$$

This reconstructs a (discrete) $3-D$ Riemanian geometry on networks
2) Gravity 3-D on Lattice: Using the previous elementary lego, we can reconstruct the Hilbert space associated with the dual graph associated with our three-dimensional geometry of space at time $t$. this is the kinematic part of the quantization. What causes geometry to change is the passage from an elementary tetrahedron to the next: it corresponds to traversing an edge of the dual graph to the discrete geometry. We deduces a first Hilbert space given by:

$$
\widetilde{\mathcal{H}}=L^{2}\left(S U(2)^{L}\right)
$$

It is necessary to take into account the gauge invariance at each vertex of the graph. Indeed the fact of choosing another moving frame must not change the result, it is therefore necessary take quotient by a product of group SU (2) on the vertex number, we then obtain the space:

$$
\left.\mathcal{H}=L^{2}\left(S U(2)^{L}\right) / S U(2)^{N}\right)
$$

3) Quantization: The quantum mechanics of the group $U(1)$ (or of the circle $S^{1}$ ) is characterized by the eigenfunctions $e^{i n x}$. which form an orthonormal system. The principle is the same to describe the quantum mechanics of $S U(2)$ : We need to find an orthogonal basis of eigenfunctions: these are Wigner matrices $D^{j}(h)_{n}^{m},-j \leq m, n \leq \leq j$ built from the irreducible representation of $S U(2)$ and labelled by an half integer $j$ in $\left\{0, \frac{1}{2}, 1, \frac{3}{2}, \ldots\right\}$ called "spin" . The operator $L^{2}=L_{i} . L_{i}$ is the Casimir operator, it acts on the Wigner matrices as follows:

$$
L^{2} D^{j}(h)=j(j+1) D^{j}(h)
$$

The eigenvalues of the Casimir operator give the quantification of the areas according to [] so

$$
A=\sqrt{j(j+1)}
$$

We can also express the volume thanks to the operator $\overrightarrow{L_{l}}$, let $l 1, \ldots l 4$ be the edges coming out of the vertex $\mathrm{n}$ : We have:

$$
V=\frac{\sqrt{2}}{3} \sqrt{\overrightarrow{L_{l_{1}}} \cdot\left(\overrightarrow{L_{l_{2}}} \times \overrightarrow{L_{l_{3}}}\right)}
$$

This formula is independent of the choice of edges because of the gauge invariance and the relation:

$$
\overrightarrow{L_{l_{1}}}+\overrightarrow{L_{l_{1}}}+\overrightarrow{L_{l_{1}}}+\overrightarrow{L_{l_{1}}}=\overrightarrow{0}
$$

There is also a quantization for the volume but this is more complicated see [21]

Geometrically, it is well known that the area of a face of the tetrahedron is given by the norm of a normal vector $\vec{E}_{i}=$ $A$. $\vec{n}$, with $\vec{n}$ normal vector to the face $i$. We then obtain $E_{i} . E_{i}=A^{2}$. We can show that $\overrightarrow{L_{i}}$ is exactly the quantization (up to a coefficient) of the vector $\vec{E}_{i}$

\section{Dynamic: Spin foams}

To summarize, we have defined operators allowing to quantify areas and volume in space. It remains to define the dynamic version, where the Hamiltonian depends on time, this will allow us to describe amplitudes that we can for 
example calculate from the path integral or using Hamiltonian mechanics. I: will now consider the temporal evolution of Spin networks: we will describe what we call spin foams.

1) Hamiltonian classical mechanic: In classical mechanics, we consider a space of configurations $\mathcal{C}$ and the evolution of a particle as a function of a universal time $q_{i}(t)$. We define the phase space $\Gamma$ by adding $p^{i}(t)$ associated momenta. In quantum mechanics, $q_{i}$ corresponds to an operator $\mid q>$, We define the Hamiltonian $H\left(q_{i}, p^{i}\right)$. Quantum dynamics is captured by the amplitudes of transitions given by the formula

$$
W\left(q_{i}^{i n}, t_{i n}, q_{i}^{f}, t_{f}\right)=\left\langle q_{i}^{f}\left|e^{\frac{i}{\hbar}\left(t_{f}-t_{i n}\right)}\right| q_{i}^{i n}\right\rangle
$$

2) Extended Hamiltonian mechanic: In relativistic mechanics (special relativity), time is no longer universal, we define an extension of the configuration and phase spaces by adding time to the space variables, we must also define a conjugate variable for the time noted $p^{t}$. the classical Hamitonian is replaced by the constrained Hamitonian $C\left(q_{a}, p^{a}\right), q_{a}=\left(q_{i}, t\right)$ the evolution is now captured by the Hamilton-Jacobi:

$$
C\left(q^{a}, \frac{\partial S\left(q^{a}\right.}{\partial q^{a}}\right)=0
$$

equation.In the Newtonian case, (privileged time) the generalized Hamiltonian is:

$$
C\left(q_{a}, p^{a}\right)=p_{t}+H\left(q^{i}, p_{i}\right)
$$

Then if $S\left(q^{i n}, t_{i n}, q^{f}, t_{f}\right)$ is a solution of I], by writing

$$
\left.\frac{\partial S\left(q^{i n}, t_{i n}, q^{f}, t_{f}\right)}{\partial q_{i}}\right)=p_{i}\left(q^{i n}, t_{i n}, q^{f}, t_{f}\right)
$$

We find the final position $q^{f}$ function of initial conditions. In the general case, the solution of the Hamilton Jacobi equation follows the same principle, however time is no longer privileged.

3) Adaptation to General relativity: Formally, we replace the couple of variable position, momenta by the couple of variable: metric 3-D, conjugate moment $\left(q_{a b}(\vec{x}), p^{a b}(\vec{x})\right)$. The Hamilton-Jacobi equation (27) becomes:

$$
G_{a b c d} \frac{\delta S\left[q_{a b}\right]}{\delta q_{a c}(\vec{x})} \frac{\delta S\left[\delta q_{a b}\right]}{\delta q_{b d}(\vec{x})}-\operatorname{det}\left(g(\vec{x}) \mathcal{R}\left(q_{a b}(\vec{x})\right)=0\right.
$$

Where $G_{a b c d}=G^{2}\left(q_{a b} q_{c d}-\frac{1}{2}\left(q_{a c} q_{b d}\right)\right.$, G gravitational constant. Set also the invariance of metric by diffeomorphism. We want to describe evolution of metric from initial: $q_{a b}^{i}$ to final $q_{a b}^{f}$ The Hamilton function is given her by: $S\left(q_{a b}^{i}, q_{a b}^{f}\right)$. we have

$$
\frac{\delta S}{\delta q_{a b}^{i}}=p^{a b}(x)=p^{a b}\left(q_{a b}^{i}, q_{a b}^{f}\right)
$$

So we obtain with initials conditions final metric. Of course here, $\mathrm{S}$ is action of Hilbert-Einstein for general relativity
Formally, $\left(q_{a b}(\vec{x}), p^{a b}(\vec{x})\right)$ become non commutative operator $q_{a b}, p^{a b}$ We can calculate "amplitudes" in quantum mechanic (with path integral form we have:

$$
W\left(q_{a b}^{f}, q_{a b}^{i}\right)=\int \mathcal{D} e^{\frac{1}{\hbar} \int \sqrt{g} \mathcal{R}}
$$

In classical limit $(\hbar \rightarrow 0)$ we find G.R:

$$
W\left(q_{a b}^{f}, q_{a b}^{i}\right)=\int \mathcal{D} e^{\frac{1}{\hbar} \int \sqrt{g} \mathcal{R}} \sim e^{\frac{1}{\hbar} \int \sqrt{g} \mathcal{R}}
$$

In general setting recall you have $\left(q_{a}, p^{a}\right)$ which satisfy classically Hamilton-Jacobi equation: $C\left(q^{a}, \frac{\partial S\left(q^{a}\right)}{\partial q^{a}}\right)=0$, given amplitudes functions $W\left(q_{a}^{f}, q_{a}^{i}\right)$ the classical Hamiltonian equation is replaced by Wheeler-Dewitt equation:

$$
C\left(q^{a}, i \hbar \frac{\partial}{\partial q^{a}}\right) W\left(q_{a}^{f}, q_{a}^{i}\right)=0
$$

In classical mechanic, with universal time, this equation generalize Shrodinger equation:

$$
\left[i \hbar \frac{\partial}{\partial t}+H\left(q^{i}, i \hbar \frac{\partial}{\partial q^{i}}\right)\right] W=0
$$

This can be transposed if we replace the coordinates by the metrics in general relativity. Now we want to solve the equation (35). the geometry at quantum level being determined by the eigenvalues of the objects to be quantified. The variables that are the initial and final 3-D metric must be replaced by their quantized forms namely spin networks: $S=\left(\Gamma, j_{l}, i_{n}\right)$ with $L$ links and $N$ nodes.

$$
W\left(q_{a b}^{f}, q_{a b}^{i}\right) \approx W\left(\left(\Gamma, j_{l}, i_{n}\right)^{\text {out }},\left(\Gamma, j_{l}, i_{n}\right)^{i n}\right)
$$

The 4-D geometry is reconstructed from the two initial and final networks. We have described the outline of the work that should be rigorous. This work has progressed a lot since then the work of different authors . In Ashekar's formalism [? ], the equation of general relativity is rewritten in equivalent form in the language of Cartan and Weil. The basic building block is the notion of mobile frame (repère mobile $=$, connection, curvature which replaces the metric field. In this language, the equations of general relativity have analogies with the Yang-Mills equations. the Hamiltonian uses the new conjugate variables which are Connection and curvature. To deal with the dynamics of quantum gravity, we must in particular study the Lie groups and algebra of $S O(3,1)$, and $S L(2 C)$. The interested reader can consult [20].

\section{INTERACTIONS BETWEEN THE DIFFERENT THEORIES}

The three theories presented briefly here, are different approaches to try to couple gravity to the standard model of particles. The starting point for quantization in non-commutative geometry and in loop quantum gravity, is geometric quantization or second quantization. Chern-Simons theory are built from Wilson loops. these are exactly the same used in the LQG. The notion of non-commutativity is also present in topological field theory, in fact the study of branes, supposed to model the physics of particles is coupled to open strings: the algebra of matrices and the group $S U(n)$ come into play 
(Chan Paton factors, K-theory of branes [3]). It is therefore tempting to be able to compare these theories. In mathematical terms it seems a vain task because the tools are noticeably different. However there are some attempts to reconcile these theories. Although not necessary for the approach of noncommutative geometry, Alain Connes in his book on Noncommutative Geometry, includes supersymmetry in his model. To compare the LQG with the supersymmetric string theory some authors [18] have constructed a quantum theory with loops in any dimensions.

\section{CONCLUSION}

In this paper we tried to describe briefly, different theories developed to understand physics beyond the Planck scale. it is a subject which is still largely misunderstood, even if progress has been made over the past thirty years. In the early $90 \mathrm{~s}$ branes were not discovered and K-Theory remained an abstract branch of algebraic topology theory. In the future several choices are possible, abandon certain tracks or try as much as possible to bring together certain theories. All the doors are open because the researchers are not close to breaking the walls of Planck...

\section{REFERENCES}

[1] M. F. Atiyah and I. M. Singer "The index of elliptic Operators on compact manifolds", in Bull. Amer. Math. Soc num.69, 1963, pp. 422-433.

[2] H. B. Lawson, M. L. Michelsohn Spin Geometry, Princeton University Press - Princeton, New Jersey, 1989.

[3] Minasian, Ruben; Moore, Gregory (1997), "K-theory and Ramond-Ramond charge", Journal of High Energy Physics, 9711 (2): 002,

[4] M.Audin Cohomologie quantique Séminaire Bourbaki, $n^{\circ}$ 807, 1995.

[5] D.A. Cox, S. Katz,Mirror symetrie and Algebraic geometry, Mathematical Surveys and Monographs V68, A.M.S 1999.

[6] S.Donaldson, P. Kronheimer,The geometry of four manifolds, Oxford University Press, 1990.

[7] P.Griffiths, J.Harris, Principle of algebraic geometry, Wiley-interscience, New York, 1978.

[8] K. Gawedzki, Lectures on Conformal field theory, in Quantum Fields and String: A Course for mathematicians, Vol 2 , A.M.S

[9] T.Friedrich Dirac Operators in Riemannian Geometry, Graduate Studies in Mathematics v.25, A.M.S 2000.

[10] K.Hori, S. Katz, A. Klemm, R. Pandharipande, R. Thomas, C. Vafa, R. Vakil, E. Zaslow ,Mirror Symmetry Clay Mathematics Monographs, Vol 1 A.M.S, 2003.

[11] M. Kontsevich, Y.Manin, Gromov-Witten Classs, Quantum Cohomology, and enumerative Geometry, Mirror symetry II, A.M.S/IP Studies in advanced Math., Vol 1 A.M.S, 1997, pp. 607-653.

[12] D.McDuff, D.Salamon, Introduction to symplectic géometry Oxford University Press, 1995.
[13] D.McDuff, D.Salamon, J-holomorphic Curves and Quantum Cohomology, Univ. Lecture series 6, A.M.S., 1994.

[14] C. Voisin Symétrie miroir, Panoramas et synthèses Numéro 2, 1996 S.M.F

[15] A. Connes Non commutative geometry, Academic Press , 1994

[16] R. Feynman Thesis A New Approach to Quantum Theory, World Scientific, 2005

[17] Philippe Durand, "Review of the Index Theorem: Applications in Mathematical Physic and Engineering Sciences," IAENG International Journal of Applied Mathematics, vol. 50, no.2, pp320-329, 2020

[18] Bodendorfer, N; Thiemann, T; Thurn, A (2013a). "New variables for classical and quantum gravity in all dimensions: I. Hamiltonian analysis". Classical and Quantum Gravity. 30 (4): 045001. arXiv:1105.3703

[19] Ashtekar A Mathematical problems of non-perturbative quantum general relativity in Gravitation and Quantizations: Proceedings of the 1992 Les Houches summer school eds Julia B and Zinn-Justin J (Elsevier, Amsterdam);

[20] Rovelli, Carlo (2011). "Zakopane lectures on loop gravity". arXiv: 1102.3660

[21] Carlo Rovelli, Quantum Gravity, Cambridge University Press (2004)

[22] Witten, Edward (1988). "Topological Quantum Field Theory". Communications in Mathematical Physics. 117 (3): 353â386.

\section{Creative Commons Attribution License 4.0 (Attribution 4.0 International, CC BY 4.0)}

This article is published under the terms of the Creative Commons Attribution License 4.0 https://creativecommons.org/licenses/by/4.0/deed.en_US 